\title{
Hot spots in fingering of exothermic autocatalytic chemical fronts
}

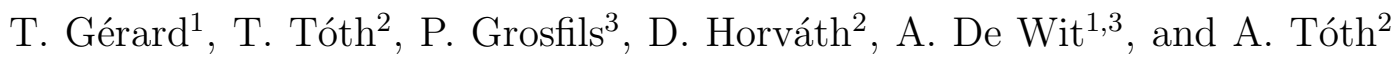 \\ ${ }^{1}$ Nonlinear Physical Chemistry Unit, Faculté des Sciences, \\ Université Libre de Bruxelles (ULB), CP231, 1050 Brussels, Belgium \\ ${ }^{2}$ Department of Physical Chemistry, University of Szeged, \\ Rerrich Béla tér 1., Szeged, H-6720, Hungary. and \\ ${ }^{3}$ Center for Nonlinear Phenomena and Complex Systems, \\ Université Libre de Bruxelles (ULB), CP231, 1050 Brussels, Belgium.
}

(Dated: June 21, 2012)

\begin{abstract}
Measurements of two-dimensional (2D) temperature fields are performed by an interferometric method during density fingering of the autocatalytic chlorite-tetrathionate $(\mathrm{CT})$ reaction in a Hele-Shaw cell. These measures confirm that, because of heat losses through the glass walls of the reactor, the temperature profile across the front is a pulse rather than a front. Moreover, the full 2D temperature field shows the presence in the reactive zone of hot spots where the temperature exceeds the maximum temperature measured in a stable planar front. We investigate here experimentally the increase of temperature in the hot spots when the composition of the reactants is varied to increase the exothermicity of the reaction. We back up these experimental observations by nonlinear simulations of a reaction-diffusion-convection model which show that the maximum temperature reached in the system depends on the intensity of convection.
\end{abstract}




\section{INTRODUCTION}

Autocatalytic reactions coupled to diffusion can generate traveling fronts of constant speed whereby products of the reaction invade and consume progressively in space and time the reactants ${ }^{1-4}$. In absence of gel, convection can deform such fronts provided an adverse density difference exists between products and reactants. The density jump between the two solutions $\Delta \rho=\rho_{\text {products }}-\rho_{\text {reactants }}$ can result from a difference in molar volume of the products compared to the reactants and from temperature changes due to the exothermicity of the reaction. In particular, convective motions appear around descending fronts as soon as $\Delta \rho>0$ i.e. for an unstable stratification of a denser solution of products lying on top of a less dense solution of reactants in the gravity field. A Rayleigh-Taylor instability whereby the denser solution sinks into the rising less dense one deforms the interface into fingers, hence the name "density fingering" also used to describe such a buoyancy-induced convective deformation of a RD front.

The resulting reaction-diffusion-convection (RDC) dynamics are typically studied experimentally in Hele-Shaw cells, i.e. quasi-bidimensional systems made of two glass plates separated by a thin gap containing the solutions in which the front propagates. A well-studied reaction is the Chlorite-Tetrathionate (CT) reaction for which Rayleigh-Taylor instability of CT fronts have been studied both experimentally and theoretically ${ }^{5-10}$. In this very exothermic reaction, the products are hotter than the reactants however, these are denser from a compositional point of view. This means that $\Delta \rho$ can be divided into two contributions: a solutal part $\Delta \rho_{s}$ which is here positive and a thermal part $\Delta \rho_{T}<0$. The CT reaction belongs thus to the family of antagonist fronts ${ }^{11}$ for which solutal and thermal effects to density changes are of opposite signs. If the total density jump $\Delta \rho$ is positive because solutal effects are dominant, then descending fronts are unstable because they feature denser products above less dense reactants. We will focus on this case in the sequel and concentrate on analyzing properties of temperature fields.

Recent experiments have started focusing on examining the specific influence of the thermal effects on the dynamics of fingers. Different techniques exist to measure the temperature field: thermocouples, infrared imaging ${ }^{12-14}$ or magnetic resonance imaging ${ }^{15,16}$ if the temperature variations are at least $1 \mathrm{~K}$. However, all these techniques allow to measure either the concentration field or the temperature field but not both of them simultaneously. The 
use of thermocolor materials is on its side intrusive ${ }^{17}$.

In this context, Grosfils et al. have recently applied an interferometric method to follow the dynamics of CT fronts which has given simultaneous access to both $2 \mathrm{D}$ concentration and temperature fields during fingering of CT fronts in Hele-Shaw cells ${ }^{18}$. Experiments show that some of the heat produced by the exothermic reaction is dissipated through the glass walls of the reactor. Because of these heat losses, the temperature profile during the propagation of the front is not a front anymore but a pulse. This modification of the temperature profile can lead to a change in the stability of fronts ${ }^{6,12,19-21}$ and in their nonlinear dynamics ${ }^{19,20,22}$. In addition, this interferometric method has also revealed that the maximum of temperature reached in the fingered front is larger than that observed in stable planar fronts. The areas where the maximum temperature is observed and that we will call "hot spots" are localized at the cusps of the fingers. Similar hot spots have also been evidenced in packed-bed reactors ${ }^{23,24}$.

It is the objective of this article to analyze in details both experimentally and numerically the properties of these hot spots in fingering of autocatalytic fronts as a function of the heat released by the reaction and of the intensity of heat losses through the walls. To do so, we first perform experiments in Hele-Shaw cells to follow by interferometry the fingering dynamics of the autocatalytic fronts in the CT reaction for increasing concentrations and hence increasing exothermicity. We next study numerically the dynamics of a related RDC model coupling Darcy's law to evolution equations for the concentration $c$ of an autocatalytic species and for the temperature $T$. We analyze the maximum temperature in the system as a function of heat release and intensity of heat losses. Numerical results compare qualitatively well with experimental findings.

The article is organized as follows: in section II, we present the experimental setup, discuss the interferometric patterns and analyze experimentally the influence of concentration changes on the properties of hot spots. Section III introduces the RDC model and describes the results of the numerical nonlinear simulations. A comparison between the two is performed. 


\section{EXPERIMENTAL}

Reagent-grade materials (Sigma, Aldrich, Reanal) were used throughout the work except for $\mathrm{NaClO}_{2}$, which was recrystallized twice. The solutions, with composition summarized in Table 1, were mixed at room temperature and injected into a $6.2 \mathrm{~cm}$ long, $3.6 \mathrm{~cm}$ large, and $1 \mathrm{~mm}$ thick reaction vessel contained in a vertically oriented so called Hele-Shaw cell, made up of two parallel transparent glass plates (Fig.1). Sodium hydroxide was added to minimize the possibility of spontaneous initiation of the autocatalytic reaction and congo red indicator was used to visualize the progression and shape of the traveling front. The autocatalytic front was initiated electrochemically by applying a $4 \mathrm{~V}$ potential difference between two parallel $\mathrm{Pt}$ wires $(0.25 \mathrm{~mm}$ in diameter $)$, which were fixed at the upper edge of the cell. The resulting downward propagating front deforms due to a Rayleigh-Taylor instability occurring because the product solution is denser than the reactant one (Fig.2). As the reaction is exothermic, the products are hotter than the reactants. If the exothermicity is sufficiently large, the thermal effect can stabilize the front ${ }^{6,11,19}$ or destabilize ascending fronts. We analyze here however situations for which the compositional effect is dominant such that descending fronts are always unstable and ascending fronts stable.

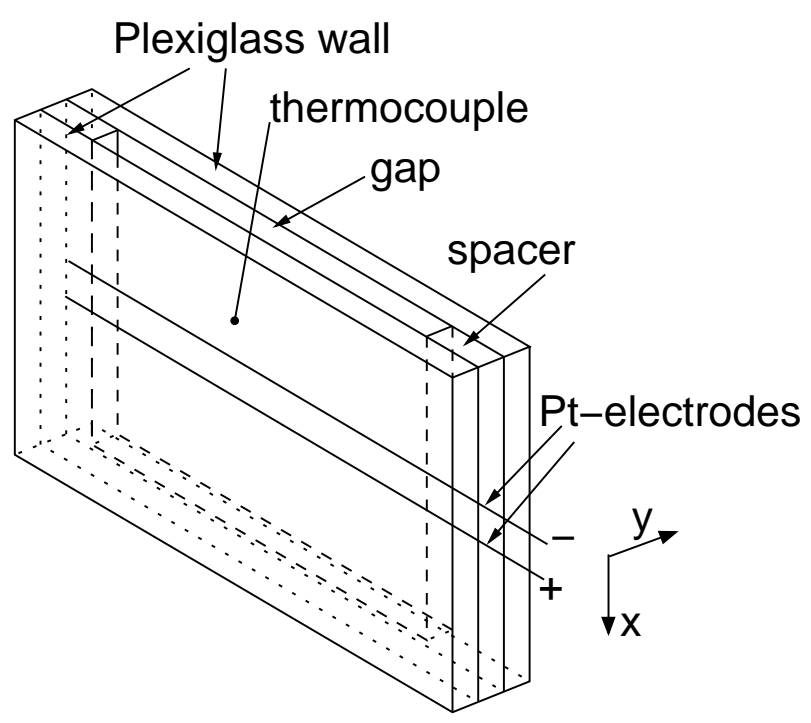

FIG. 1. Sketch of the system. 
TABLE I. Composition of reactant solution for three different sets of experiments: $c_{1}, c_{2}$ and $c_{3}$.

\begin{tabular}{l|ccc}
\hline & $c_{1}$ & $c_{2}$ & $c_{3}$ \\
\hline \hline$\left[\mathrm{K}_{2} \mathrm{~S}_{4} \mathrm{O}_{6}\right] / \mathrm{mM}$ & 4.54 & 5 & 7.5 \\
{$\left[\mathrm{NaClO}_{2}\right] / \mathrm{mM}$} & 18.2 & 20 & 30 \\
{$[\mathrm{NaOH}] / \mathrm{mM}$} & 9.1 & 5.0 & 5.0 \\
{$[$ Congo red $] / \mathrm{mM}$} & 0.574 & 0.574 & 0.574 \\
\hline
\end{tabular}

\section{A. Optical measurement method}

The temporal evolution of two-dimensional temperature and concentration fields is obtained using a nonintrusive optical technique that combines digital interferometry and transparency measurement ${ }^{18}$. In this mixed technique, a direct transparency image is recorded that reveals the concentration field thanks to the change in color of the congo red indicator. The result is an image, shown in Fig.2a, whose color changes from red (grey in Fig.2a) in the reactant to blue (black in Fig.2a) in the acidic product. At the same time, digital interferometry is used to record a two-dimensional map of the optical path difference, that is the difference of the refractive index integrated along different optical paths. The first path, that goes through the Hele-Shaw cell, contains a contribution from the refractive index of the solution inside the cell and from the cell itself, that is the two glass plates. Only the refractive index of the air contributes to the second optical path. The difference between the two optical paths is encoded in the bending of the fringe shift of an interferogram (see Fig.2b) and can be retrieved by a Fourier method ${ }^{26,27}$. The result is an image of the optical phase shift. In order to eliminate the shift due to the glass of the cell, a reference image, that here corresponds to a homogeneous cell without any front, is substracted from the optical phase image. This gives the corrected phase image (Fig.2c) whose intensity $\Delta \phi(x, y)$, is proportional to $\Delta \bar{n}(x, y)$, the difference between the refractive index of the solution and a constant background value

$$
\Delta \phi(x, y)=\frac{2 \pi L_{z}}{\lambda} \Delta \bar{n}(x, y)
$$

Here the bar denotes an average over the depth of the sample, $L_{z}$ is the thickness of the gap in the Hele-Shaw cell, and $\lambda$ is the wavelength of the light $(\lambda=633 \mathrm{~nm})$.

Usually, experiments are performed at constant temperature such that the variations of 
refractive index inside the cell are due to concentration variations only. In that case, the concentration field is readily available from the phase image when assuming a linear relation between the refractive index and the concentration. In our experiments on the contrary, the exothermicity of the reaction implies that temperature variations also modify the refractive index and hence

$$
\Delta \phi(x, y)=\frac{2 \pi L_{z}}{\lambda}\left(\frac{\partial n}{\partial c} \Delta c(x, y)+\frac{\partial n}{\partial T} \Delta T(x, y)\right) .
$$

In this relationship, $\Delta \phi$ is the corrected phase therefore, $\Delta T(x, y)$ and $\Delta c(x, y)$ may be interpreted as differences between the value of the temperature and concentration fields at the location $(x, y)$ and the values of these fields at a fixed reference location inside the cell. If one assumes that the solutions are dilute, then the variation of the refractive index is linear with the temperature and the concentration, so that $\partial n / \partial c$ and $\partial n / \partial T$ are constants, and Eq. (2) becomes

$$
\Delta \phi(x, y)=a_{1} \Delta c(x, y)+a_{2} \Delta T(x, y)
$$

where the $a_{i}$ 's are unknown constants. To obtain $\Delta T$ from this relationship one uses the transparence imaging of the sample, taken at a fixed temperature, which gives a measurement of the absorption of the light by the fluid. The absorption is assumed to be independent on the temperature and to depend on the concentration only. The transparence imaging gives an independent measurement of the concentration field (see Fig.1a) that can be used to reconstruct the temperature field once the constants $a_{i}$ 's are known, via the relationship

$$
a_{2} \Delta T(x, y)=\Delta \phi(x, y)-a_{1} \Delta c(x, y)
$$

which, in practice, is computed as the difference between the intensities of two images. To determine the values of $a_{1}$ and $a_{2}$, the temperature is measured by a thermocouple at two positions located respectively far ahead and far behind the front, that correspond to $\Delta c=0$ in the fresh reactant zone and $\Delta c=1$ in the product respectively. This information, together with the phase field, is used to calibrate the values of the $a_{i}$ 's so that the value of the temperature obtained by the optical apparatus corresponds, along the measured line, to the value obtained by the thermocouple. More specifically, the thermocouple gives a temporal evolution of the temperature at a fixed position however, as the front propagates with a constant velocity, the profile in time is easily converted to a temperature profile in space. The calibration step is illustrated in Fig.3, that displays the temperature profile 
as measured by the thermocouple together with the temperature profile obtained by the optical technique, after calibration. After this calibration step, the full 2D temperature field can then be recovered (Fig.4). This method gives a measurement of the two-dimensional temperature field with a precision of about $0.05^{\circ} \mathrm{C}$.
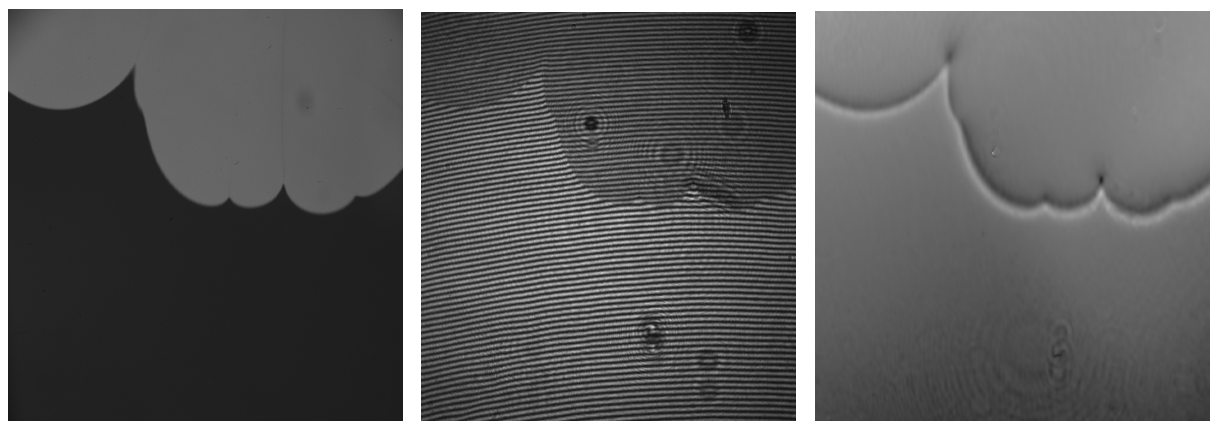

FIG. 2. Fingering of a chemical front visualized as an absorption image (left), an interferometric image (middle), and a corrected optical phase image (right).

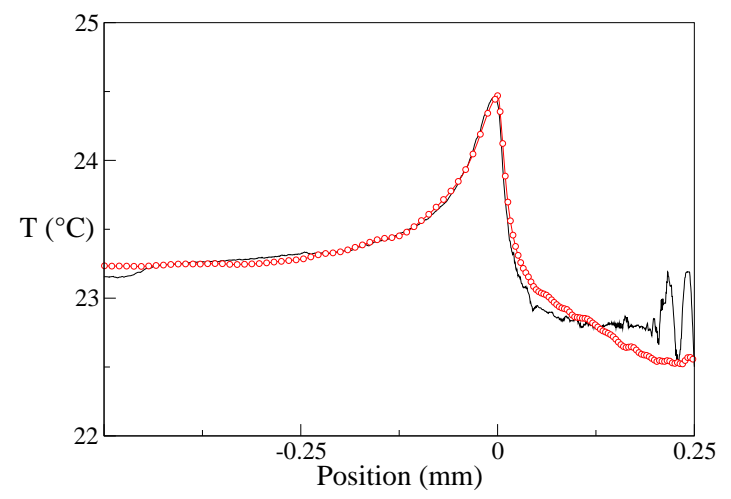

FIG. 3. Temperature profile across the chemical front obtained by the optical technique (cercles) and via the thermocouples (line). The view is perpendicular to the glass plates i.e. in a $(x, y)$ plane as shown on Fig.1.

\section{B. Experimental results}

When chemical concentrations are increased, thermal effects are increased. The chlorite oxidation of tetrathionate is indeed a highly exothermic reaction with an enthalpy of reaction 

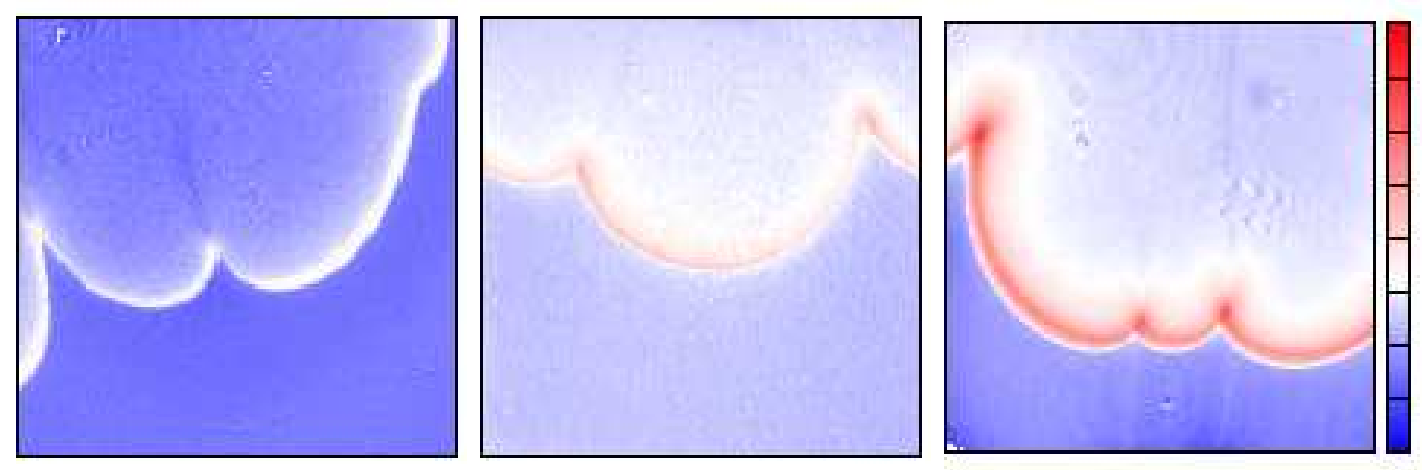

FIG. 4. Experimental 2D temperature field at concentration $c_{1}, c_{2}$, and $c_{3}$ from left to right, shown on the same temperature scale. The temperature maximum in the front increases with the exothermicity of the recipe $\left(c_{3}>c_{2}>c_{1}\right)$. The hottest zones are located at the cusps of the fingered zone.

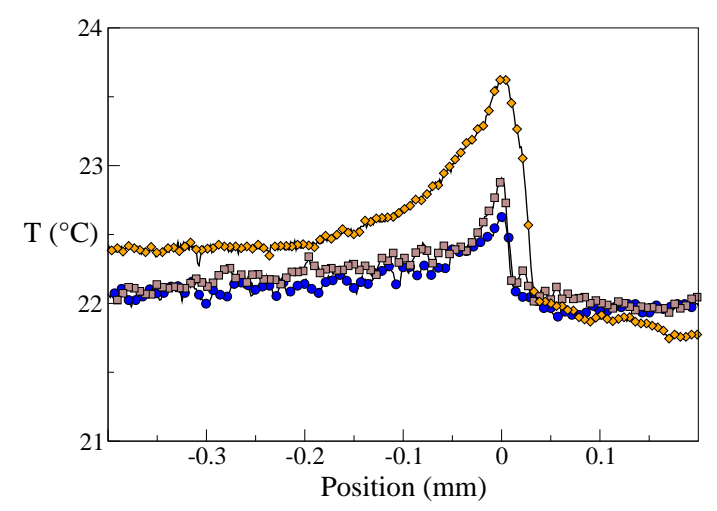

FIG. 5. Temperature profile across the front for the chemicals at concentration c1 (circles), c2 (squares), and c3 (diamonds).

of -3960 kJ/mol. We have here experimentally analyzed fingering in a vertical Hele-Shaw cell of downward propagating CT fronts for various compositions (see Table 1) chosen to increase progressively the exothermicity of the reaction. The $c_{1}$ experimental conditions would yield an adiabatic temperature increase of $2.1 \mathrm{~K}$ given by $\Delta T_{a}=\Delta_{r} H\left[\mathrm{~S}_{4} \mathrm{O}_{6}^{2-}\right]_{0} /\left(2 \rho c_{p}\right)$ where the density $\rho$ and the specific heat capacity $c_{p}$ are approximated by the values for water, i.e., $\rho=1 \mathrm{~g} / \mathrm{cm}^{3}$ and $c_{p}=4.19 \mathrm{~J} /(\mathrm{g} \mathrm{K})$. The addition of sodium hydroxide prevents spontaneous self-initiation through the removal of the hydrogen ion produced in the reaction. Since 
this neutralization reaction is exothermic as well with $\Delta_{r} H=-55 \mathrm{~kJ} / \mathrm{mol}$, the adiabatic temperature rise should be corrected by taking into account the concentration of sodium hydroxide. The adiabatic temperature increases therefore to $2.3 \mathrm{~K}$ for the $c_{1}$ conditions with the concentration of sodium hydroxide at $9.1 \mathrm{mM}$. By decreasing the amount of sodium hydroxide for the $c_{2}$ experimental conditions, we can increase the velocity of front propagation, however the change in the adiabatic temperature rise is negligible $\left(\Delta T_{a}=2.4 \mathrm{~K}\right)$, since it is mainly determined by the reactants. This is what we observe at greater reactant concentrations at constant ratio (see $c_{3}$ conditions in Table I), the adiabatic temperature rise $T_{a}$ $=3.6 \mathrm{~K}$ is significantly greater.

From the overall adiabatic temperature change, the maximal density decrease that can exist across the front due to the temperature change can be calculated as $\Delta \rho_{T}=(d \rho / d T) \times$ $\Delta T_{a}$ where $d \rho / d T$ can be estimated from the temperature dependence of water density. When the chemical concentrations are increased, the adiabatic temperature rise and hence the thermal density decrease is increased significantly. As a consequence the heat release increases which can be witnessed in the local measurement of the temperature using a thermocouple where the amplitude of the maximum temperature increases (Fig.5). An important information obtained from this temperature profile is that heat losses are found to be important as the temperature profile is not a front but a pulse ${ }^{20}$. This is due to the fact that the heat produced by the reaction inside the front escapes in time through the glass walls. The resulting 2D dynamics shown on Fig.4 confirms this as the reactants are at room temperature, the products are hotter but yet the hottest location is obtained at the rim of the front where the reaction takes place. The products behind the front are cooled down because of heat escape though the walls of the reactor.

A comparison between the maximum temperature measured locally along a cross-section (Fig.5) and that obtained by interferometry on the full 2D temperature field (Fig.4) shows that the cross-section has missed the largest value of temperature accessed into the system. Indeed, looking at the experimental figures for the recipe $c_{3}$, one can see that the maximum of temperature of a stable front (Fig.5, $T_{\max }<23.75^{\circ} \mathrm{C}$ ) is smaller than in an unstable fingering front (Fig.4, $T_{\max } \approx 25^{\circ} \mathrm{C}$ ) as visible on the temperature scales.

This is explained by the fact that the locations of largest temperature (that we here call "hot spots") are at the cusps of the fingered front as seen on Fig.3. The spatial extent of these hot spots is small and the probability that these hot zones pass exactly at the location 
of the thermocouple is small. In effect, we have checked that the temperature recorded by the thermocouple and shown on Fig.2 has been indeed measured in the middle of a finger where the temperature is not maximum. The interferometric method shows here its whole interest which is to be able to evidence the presence of hot spots in the cusp of different fingers ${ }^{18}$. A comparison in Fig.3 of the 2D temperature fields obtained for various concentrations shows that the largest temperature reached increases with the increase of the exothermicity of the reaction when concentrations are varied. To understand the origin and properties of these hot spots and whether the maximum temperature measured in the presence of convection is larger than the adiabatic maximum temperature reached in stirred systems, we now turn to numerical simulations of the problem.

\section{SIMULATIONS}

\section{A. Model}

As experiments show no evidence of convection in the gap direction, the experimental setup is numerically modeled as a two-dimensional system of length $L_{x}$ and width $L_{y}$, oriented respectively along the vertical $x$ and horizontal $y$ axes. Gravity $\underline{g}$ is pointing in the positive $x$ direction. We numerically investigate the two-dimensional dynamics by solving the following system of nonlinear equations ${ }^{20}$

$$
\begin{gathered}
\underline{\nabla} \cdot \underline{u}=0, \\
\underline{\nabla} p=-\frac{\mu}{\kappa} \underline{u}+\rho(c, T) \underline{g}, \\
\frac{\partial c}{\partial t}+\underline{u} \cdot \underline{\nabla} c=D \nabla^{2} c+f(c), \\
\rho_{0} c_{p}\left[\frac{\partial T}{\partial t}+\underline{u} \cdot \underline{\nabla} T\right]=\kappa_{T} \nabla^{2} T+|\Delta H| f(c)-\alpha\left(T-T_{0}\right) .
\end{gathered}
$$

expressing the incompressibility of the fluid (5) and the evolution of the fluid velocity $\underline{u}$ through Darcy's equation (6) where the permeability $\kappa$ depends on the gapwidth $L_{z}$ between the plates as $\kappa=L_{z}^{2} / 12$ and $\mu$ is the viscosity of the fluid. Eq.(7) and (8) are the reactiondiffusion-convection evolution equations for the autocatalytic product concentration $c$ and 
for the temperature $T$ released by the reaction respectively where $D$ is the molecular diffusion coefficient, $T_{0}$ is the temperature of the reactants, $\rho_{0}$ the density of the solvent (water here), $c_{p}$ the constant pressure heat capacity, $\kappa_{T}$ the thermal conductivity, $\Delta H$ the enthalpy of reaction and $f(c)$ the reaction kinetics while $\alpha$ is Newton's heat loss coefficient.

As we work with diluted solutions, the density $\rho$ is assumed to depend linearly on $c$ and $T$ as

$$
\rho(c, T)=\rho_{0}\left[1+\Delta \rho_{c}+\Delta \rho_{T}\right]
$$

where $\rho_{0}$ is the density of water and $\Delta \rho_{c}, \Delta \rho_{T}$ are the density jumps between the reactants and the products due to compositional and thermal changes respectively. These quantities are defined as $\Delta \rho_{c}=\alpha_{c}\left(c-c_{0}\right)$ and $\Delta \rho_{T}=\alpha_{T}\left(T-T_{0}\right)$ where $\alpha_{c}=\frac{1}{\rho_{0}} \frac{\partial \rho}{\partial c}$ and $\alpha_{T}=\frac{1}{\rho_{0}} \frac{\partial \rho}{\partial T}$ are the solutal and thermal expansion coefficients respectively. $c_{o}=0$ is the concentration of products in the reactants and $T_{0}$ is the initial temperature of the reactants. The jumps of concentration and of temperature across the front are respectively $\Delta c=c_{1}-c_{0}$ and $\Delta T=\left(T_{1}-T_{o}\right)=-\Delta H \Delta c / \rho_{0} c_{p}$ where $c_{1}$ and $T_{1}$ are the concentration and temperature of the products in absence of heat losses i.e. the temperature change that would be measured in an isolated stirred reactor for instance. The temperature jump in our experiments is relatively small and hence the kinetic constant $k$, the diffusion coefficient $D$ of the product and the thermal conductivity $\kappa_{T}$ are considered constant.

To obtain dimensionless equations, we define $c^{\prime}=c / \Delta c, T^{\prime}=\left(T-T_{o}\right) / \Delta T$. The characteristic chemical time $\tau=\left(k \Delta c^{2}\right)^{-1}$ needed to convert the reactant into product where $k$ is the kinetic constant is used to define the length $L_{h}=\sqrt{D \tau}$ and velocity $U=\sqrt{D / \tau}$ scales. We next incorporate the constant hydrostatic pressure term $\rho_{0} \underline{g}$ into a renormalized gradient of pressure term and introduce the Lewis number $L e=\frac{\kappa_{T}}{\rho_{0} C_{P} D}$ and $\alpha^{\prime}=\alpha \tau$. We obtain the following system of equations (after removing the primes) ${ }^{28}$

$$
\begin{gathered}
\underline{\nabla} \cdot \underline{u}=0, \\
\underline{\nabla p}=-\underline{u}+\left(R_{c} c+R_{T} T\right) \underline{e}_{x}, \\
\frac{\partial c}{\partial t}+\underline{u} \cdot \underline{\nabla} c=\nabla^{2} c+f(c),
\end{gathered}
$$




$$
\frac{\partial T}{\partial t}+\underline{u} \cdot \underline{\nabla} T=L e \nabla^{2} T+f(c)-\alpha T
$$

where $\underline{e}_{x}$ is the unit vector pointing downwards along $x$. As a classical dimensionless kinetic scheme providing traveling fronts when coupled to diffusion ${ }^{25}$, we take $f(c)=c^{2}(1-c)$. The solutal and thermal Rayleigh numbers $R_{c}$ and $R_{T}$ are defined as

$$
R_{c}=\frac{i \kappa \alpha_{c} \triangle c}{\nu U}, R_{T}=\frac{i \kappa g \alpha_{T} \triangle T}{\nu U}
$$

where $i=-1$ for descending fronts and $i=1$ for ascending fronts. A positive $R_{c}$ corresponds to a buoyantly unstable situation of a solute rich and hence denser solution on top of a less dense one (Rayleigh-Taylor instability) while a positive $R_{T}$ corresponds to an unstable heating of the solution from below (Rayleigh-Bénard instability). If $L e \neq 1$, double-diffusive instabilities can be obtained if $R_{c}$ and $R_{T}$ have opposite signs ${ }^{11,29}$ while chemically-driven instabilities can develop due to a spatially dependent reaction rate even if $R_{c}, R_{T}$ are both negative ${ }^{11,28}$. We focus thus here on situations where $R_{c}$ and $R_{T}$ have opposite signs as is the case for the CT reaction but such that $R_{c}>\left|R_{T}\right|$ in order to have density fingering (solutal effects win) on the descending front and a stable ascending one. Linear stability analysis has shown that in this quadrant of the $\left(R_{c}, R_{T}\right)$ plane, direct RT exists for $R_{c}>\left|R_{T}\right|$ and double diffusion effects destabilize almost all the rest of the quadrant. Moreover, recent studies showed that heat losses tend to destabilize descending fronts of this quadrant ${ }^{21}$. Indeed, in this part of the $\left(R_{c}, R_{T}\right)$ plane, temperature is stabilizing fronts propagating downwards. As heat losses decrease the temperature difference between products and reactants, the density jump across the front is larger than in absence of heat losses and fronts are thus destabilized by heat losses in this quadrant of the $\left(R_{c}, R_{T}\right)$ plane.

A typical value of $R_{c}$ corresponding to the experimental conditions ${ }^{30}$ is around 2.4 if we use $g=9.81 \mathrm{~m} / \mathrm{s}^{2}$ and $\Delta \rho=3 \times 10^{-4}$. The gap width $L_{z}$ is $1 \mathrm{~mm}$ giving a permeability $\kappa=1 \mathrm{~mm}^{2} / 12$. The solution viscosity is $\mu=1 \mathrm{cP}$, and $U \sim 0.01 \mathrm{~mm} / \mathrm{s}$. To calculate the thermal contribution $R_{T}$, we note that the temperature increase $\Delta T$ is around $1.5 \mathrm{~K}$ in the experiments ${ }^{7}$ considered, leading to $\Delta \rho_{T}=-4 \times 10^{-4}$. These data suggests values of $R_{T} \sim 3.2$. In the numerical simulations, the solutal and thermal Rayleigh numbers are varied in the range 1-5 to cover typical experimental conditions. 


\section{B. Nonlinear simulations}

The nonlinear simulations are performed using a pseudo-spectral numerical scheme ${ }^{31}$ on a rectangular $2 \mathrm{D}$ system of length $L_{x}=1024$ and width $L_{y}=512$ with periodic boundary conditions. To be able to follow simultaneously both the buoyantly unstable descending and the stable ascending fronts, the initial condition consists in a system filled with reactants at room temperature $(c=0, T=0)$ while a band of autocatalytic hot product $(c=1, T=1)$ is added both on the top and bottom of the system. Specifically, the initial concentration field corresponds to two back to back step functions with noise added in the transition zones. The initial temperature field is identical to the concentration field i.e. $T_{0}(x, y)=c_{0}(x, y)$. Walls of the Hele-Shaw cell are dissipating heat out of the experimental system, leading to a pulse of temperature. In the model, these pulses are observed for $\alpha \neq 0$. We fix here Newton's coefficient to $\alpha=0.1$, an intermediate value giving temperature pulses alike those measured experimentally. In experiments, the change in initial concentrations of $\mathrm{K}_{2} \mathrm{~S}_{2} \mathrm{O}_{4}$, $\mathrm{NaClO}_{2}$ and of $\mathrm{NaOH}$ is affecting the heat production of the reaction and modifying the intensity of the temperature profile (Fig.5). $R_{c}$ has been fixed to $R_{c}=2$ while we increase $R_{T}$ from -1 to -4 to consider a change in heat generation. The Lewis number is fixed to $L e=10$ as heat diffuses faster than chemical species. This relatively small value is realistic for the CT reaction because the autocatalytic species, i.e. protons, diffuse quite fast.

\section{Reaction-diffusion profiles}

Before studying convective fingering dynamics, it is useful to recall the properties of the underlying reaction-diffusion system. Integrating model (10-13) with $R_{c}=R_{T}=0$ gives the one-dimensional RD concentration and temperature profiles. The solution for the concentration profile can be obtained analytically and is a well-known ${ }^{2}$ hyperbolic tangent profile traveling at a constant speed $v$. If $L e=1$ and $\alpha=0$, the evolution equation for temperature and concentration are exactly the same and, as a consequence, the temperature front exactly superposes the concentration one (Fig.6). In absence of heat losses $(\alpha \neq 0)$ but $L e>1$, the temperature distribution cannot be computed analytically any longer and is therefore obtained numerically. We see on Fig.6 that the thermal front extends therefore ahead of the reaction front and heat is heating part of the fresh reactant solution. The 
larger Le the more extended the temperature distribution. Heat losses $(\alpha \neq 0)$ also affect the temperature profile by changing it from a front to a pulse. The maximum of temperature is located at the point where the reaction is the strongest, approximatively at $z=0$ where $z=x-v t$ is the coordinate traveling at the front speed $v$. The larger $\alpha$ the less extended the temperature distribution and the smaller the intensity of the maximum of temperature.

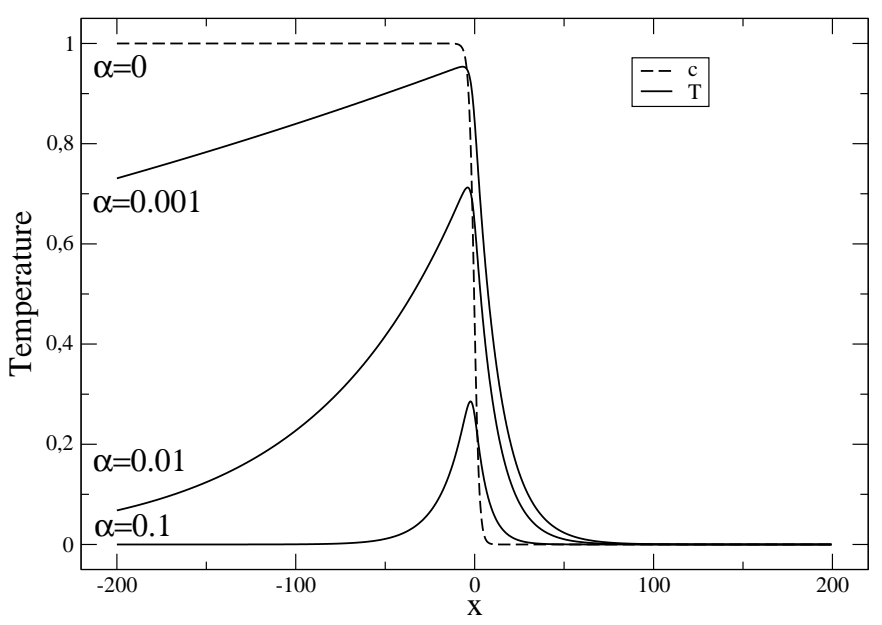

FIG. 6. Reaction-diffusion concentration and temperature profiles for $L e=1$ (dashed curve) and temperature profiles for $L e=10$ and various values of $\alpha$ (full curves) in a frame moving at the RD speed $v$.

\section{Hot spots}

Experiments have shown that the cusps of the fingers are warmer than the rest of the front. We performed non-linear simulations to determine the source of such hot spots during the fingering of an autocatalytic front. The advantage of simulations is to be able to measure simultaneously temperature and concentration profiles in both unstable descending and stable ascending fronts. Fig.7 shows concentration and temperature fields for a typical set of parameters such that the ascending front is stable while the descending one is unstable and features fingers with a given wavelength. In the course of time, some of those fingers merge and a general coarsening trend is obtained. The related temperature field shows 

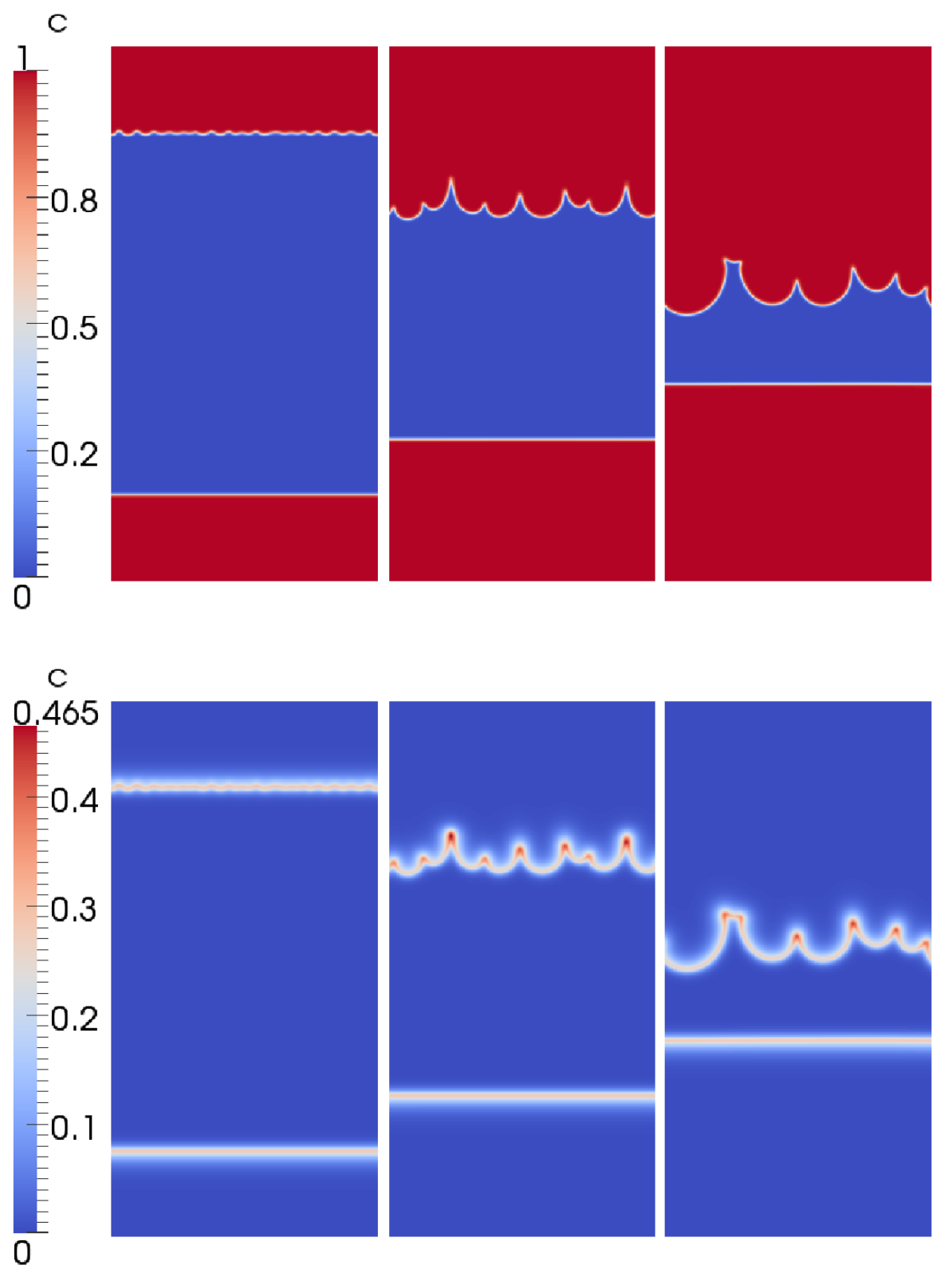

FIG. 7. Nonlinear dynamics of concentration (top) and temperature (bottom) for $R_{c}=2, R_{T}=-2$, $L e=10$ and $\alpha=0.1$ at times $\mathrm{t}=150,300,450$ (from left to right).

that quickly the initial temperature step profile is modified into a pulse that propagates at constant speed around the concentration front. Once fingering starts, hot spots are observed in the cusps of the fingers as bright area i.e. zones where locally the temperature is larger than in the stable planar front.

To characterize the intensity of temperature reached in these hot zones, we compare in 


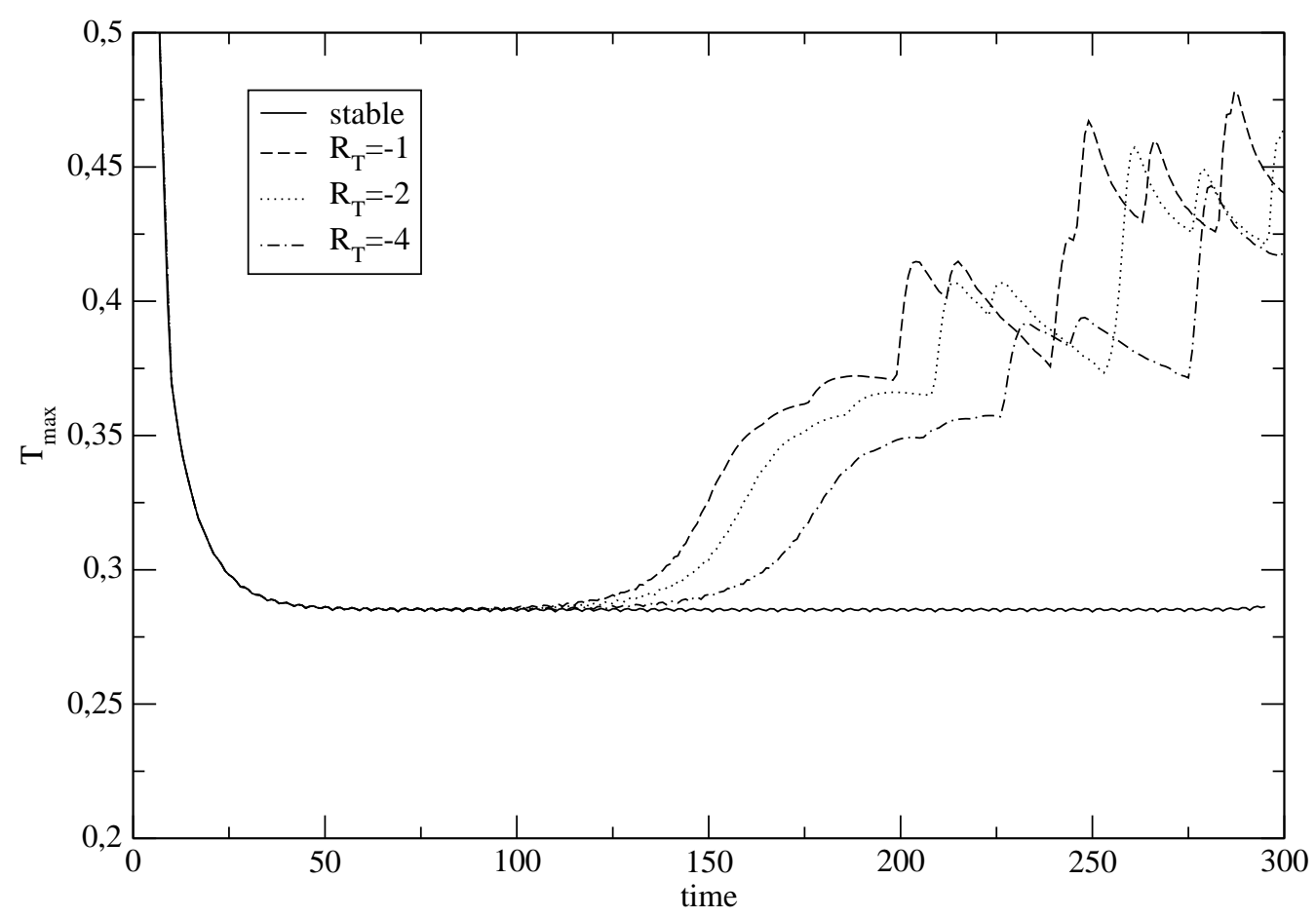

FIG. 8. Maximum temperature reached during the dynamics as a function of time for $R_{c}=2$, $\alpha=0.1$ and various values of $R_{T}$.

time the maximum of the temperature $T_{\max }$ reached in both the ascending and descending fronts (Fig.8). At the beginning, $T_{\max }=1$ in the initial condition. The presence of heat losses rapidly transforms the initial temperature step function into a pulse in which the maximum temperature is smaller than $T=1$. For the specific values of parameters $R_{c}=2$ and $\alpha=0.1$ studied in Fig.8, $T_{\max }$ equals 0.28 in the stable ascending front. For the downward moving front, this value is observed as long as the front remains planar. Once the hydrodynamic instability sets in (around 100 units of time) and fingering is observed, $T_{\text {max }}$ increases in the fingered front. The larger $\left|R_{T}\right|$ at a fixed positive $R_{c}$, the larger the stabilizing thermal component and hence the later the fingering witnessed by an increase of $T_{\max }$ above 0.28 is obtained. The various bumps observed later in the temporal evolution of $T_{\max }$ relate to merging of some fingers. As hot zones exist in the cusps of fingers, these areas disappear when two fingers merge which explains the sudden decrease in $T_{\text {max }}$ during 

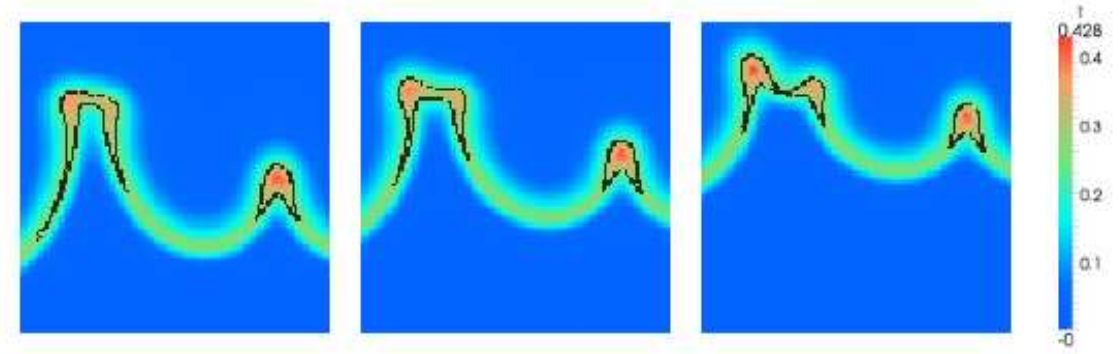

FIG. 9. 2D temperature field for descending fronts and $R_{c}=2, \alpha=0.1, \mathrm{Le}=10$ and $R_{T}=-1,-2$ and -4 from left to right.

finger reorganization. We observe on Fig.7 that the temperature obtained in cusps between two large fingers is larger than that measured in cusps in the vicinity of small perturbations.

Moreover, as the temperature is larger into hot spots, the local density is there slightly smaller than in the rest of the front. Hot spots tend thus to rise against the gravity. One can see on Fig.9 that an increase of the absolute value of $R_{T}$ leads to the focalisation of the heat in the hot spot. Indeed, the area delimited by the black line, which is the isotemperature curve where $T=0.27$, decreases in size and is slightly moving upwards. Fig.9 also shows that the temperature at the tip of the fingers is smaller than in a stable front, i.e. it doesn't reach $T=0.27$ in this case. Fingered fronts feature thus tips that are colder and cusps that are hotter than the maximum temperature reached in planar fronts. We can therefore conclude that, in such curved fronts, heat is transported from the tip of the fingers to their cusps. A superposition of the $2 \mathrm{D}$ temperature field with isovelocity lines (Fig.10) shows that the minima of temperature in the tip and the maxima in the cusps concide with the zones of descending and ascending fluid motions respectively indicating that the convection is diluting heat at the tip and concentrating it at the cusps.

\section{Comparison with experiments}

Quantitative comparison with experiments is difficult here because, first of all, we do not exactly know how the change of concentrations of the different species affects the solutal and thermal contributions to density and hence $R_{c}$ and $R_{T}$. The Rayleigh numbers we use are however in the range of experimental values. The second reason is that we use a general third order kinetics which coupled to diffusion produces a front while the CT reaction is best 


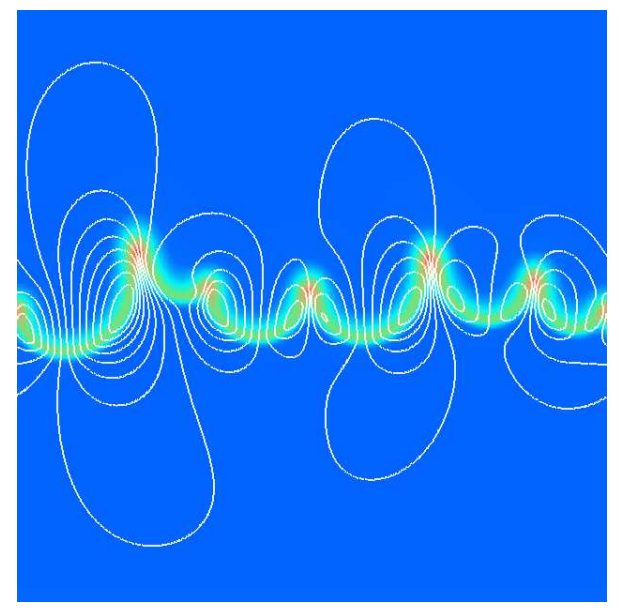

FIG. 10. 2D temperature field for a descending front $\left(R_{c}=2, \alpha=0.1, \mathrm{Le}=10\right.$ and $R_{T}=-2$, $)$, at $\mathrm{t}=400$ with isovelocity lines.

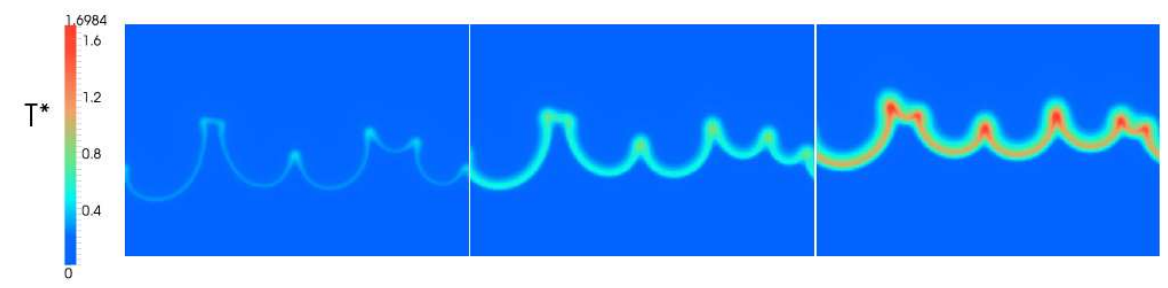

FIG. 11. 2D rescaled temperature $T^{*}=R_{T} T$ field for descending fronts and $R_{c}=2, \alpha=0.1$, $\mathrm{Le}=10$ and $R_{T}=-1,-2$ and -4 from left to right.

modeled by a one variable fourth-order kinetics ${ }^{19}$. Thirdly, the flow field is here modeled by Darcy's law valid only for very small Hele-Shaw gap width. 3D effects can possibly also affect the quantitative values of the fingered dynamics ${ }^{32}$.

Nevertheless, we can try to understand the qualitative impact of changes in concentrations in the dynamics. In effect, increasing the concentration of the reactants increases the exothermicity and thus the heat release. It certainly has an influence on the solutal component to density and thus on $R_{c}$ but, as we don't know to what extent and also in order to isolate the thermal effect, let us keep $R_{c}$ constant and compare the intensity of the hot spots for increasing heat effects i.e. decreasing negative $R_{T}$. In dimensionless scales such as in Fig.9, we cannot appreciate the change in the comparison because temperature is for each case varying between between 0 and 1 as it is normalized by the temperature jump 
across the front. We plot therefore on Fig.11 the dimensionless temperature multiplied by $R_{T}$ which shows that, when the exothermicity is increased, the maximal value reached in the temperature is indeed increasing and the cusps of the fingers become hotter and hotter. These numerical simulations compare qualitatively well with the experimental results of Fig.3.

\section{CONCLUSION}

In the experiments the results obtained from the interferometric method clearly demonstrate that hot spots develop at the trailing segments of the reaction front. These cusps joining the fingers not only represent local maxima in temperature along the reaction front but also have higher temperature than that in an adiabatic case in a homogeneous system. The scenario is in strong contrast to that associated with the hot spots in premixed laminar flames: in that system due to the interaction of reaction kinetics with thermal diffusivity, hot spots arise in the leading segments of the front surrounded by the fresh reactants, while the cusps are colder because they are short of fuel and hence heat production is at minimum. ${ }^{33}$ In our convective system, however, the unefficient heat dissipation from the cusps due to the flow pattern dominates, leading to temperature maxima at these locations.

Numerical simulations of the problem, even if they cannot be compared quantitatively with the experimental data, enlighten nevertheless the problem. They indeed show that hot spots in the fingered front correspond to zones where the local temperature exceeds the maximal temperature reached in a flat stable front. In particular, the simulations have evidenced the fact that the tip of the fingers are colder while their cusps are hotter than the maximal temperature reached in a flat front. These temperature profiles follow the velocity profiles suggesting that convection dilutes heat at the tips and concentrate it at the cusps. Moreover, it appears logical that the hot spots which are also the less dense zones are rising in the gravity field and are therefore located at the cusps which are the highest zones of the fingered front. Increase in the exothermicity of the problem by increasing the negative value of $R_{T}$ provides the same effect as in the experiments, i.e. an increase of the temperature in the hot spots.

Future perspectives of this work are twofold. First, if better quantitative agreement with experiments is the goal, we will need to obtain more information on the respective changes 
in the solutal and thermal components to the density jump across the front as a function of concentrations. This will imply to make measurements of densities for various compositions of the reactants both at room temperature and at $3^{\circ} \mathrm{C}$ where thermal effects are cancelled. The modeling could also be adapted to take the real kinetics into account and possible 3D effects.

Second, from a theoretical perspective, more simulations need to be done to understand the relative role of curvature and convection on the properties of hot spots. Indeed, preliminary simulations made with $R_{T}=0$ show that hot spots exist even when thermal effects do not influence the density. It is logical in a way that forced convection modifies the distribution of temperature which behaves as a passive scalar for $R_{T}=0$. Nevertheless, it remains to understand why the temperature can in that case exceed the maximal temperature obtained in a flat front.

Acknowledgements This work was financially supported by the Hungarian Scientific Research Fund (OTKA K72365) and the European Space Agency (ESTEC 4000102255/11/NL/KML). A.D. acknowledges financial support from FRS-FNRS, Prodex and ESA. P.G. thanks FRSFNRS for financial support under contract C-Net NR/FVH972. T.G. has benefitted from a fellowship of FRIA (Belgium) which is gratefully acknowledged.

1 V. Mendez, S. Fedotov, W. Horsthemke, Reaction transport systems, Springer Verlag, (2010).

2 A. Saul, K. Showalter, "Propagating reaction-diffusion fronts". In Oscillations and Travelling Waves in Chemical Systems, R. Fields and M. Burger Eds, 1985.

3 I.R. Epstein, J.A. Pojman, An Introduction to Nonlinear Dynamics: Oscillations, Waves, Patterns and Chaos, Oxford University Press, New York (1998).

${ }^{4}$ K. Showalter, Nonlinear Science Today 4, 3 (1995).

5 D. Horváth, T. Bánsági Jr., Á. Tóth, J. Chem. Phys. 117, 4399 (2002).

6 T. Bánsági Jr., D. Horváth, Á. Tóth, J. Yang, S. Kalliadasis, and A. De Wit, Phys. Rev. E 68, 055301 (2003).

7 T. Bánsági Jr., D. Horváth, Á. Tóth, Phys. Rev. E 68, 026303 (2003).

8 T. Bánsági Jr., D. Horváth, Á. Tóth, Chem. Phys. Lett. 384, 153 (2004). 
9 G. G. Casado, L. Tofaletti, D. Müller, A. DOnofrio, J. Chem. Phys. 126, 114502 (2007).

10 L. Macias, D. Müller, A. D’Onofrio, Phys. Rev. Lett. 102, 694501 (2009).

11 J. D'Hernoncourt, A. Zebib, A. De Wit, Chaos 17, 013109 (2007).

12 J. Martin, N. Rakotomalala, L. Talon, D. Salin, Phys. Rev. E 80, 055101 (2009).

13 M. Böckmann, B. Hess, S.C. Müller, Phys. Rev. E 53, 5498 (1996).

14 I.P. Nagy, A. Keresztessy, J.A. Pojman, J. Phys. Chem 99, 5385 (1995).

15 M. Britton, J. Phys. Chem. A 110, 5075 (2006).

16 V. Zhivonitko, I. Koptyug, R. Sagdeev, J. Phys. Chem. A 111, 4122 (2007).

17 I.P. Nagy, J.A. Pojman, Chem. Phys. Lett. 200, 147 (1992).

18 P. Grosfils, F. Dubois, C. Yourassowsky, and A. De Wit, Phys. Rev. E 79, 017301 (2009).

19 S. Kalliadasis, J. Yang, A. De Wit, Phys. Fluids 16, 1395 (2004).

20 J. D'Hernoncourt, A. De Wit, Physica D 239, 819 (2010).

21 T. Gérard, A. De Wit, Wave Motion, 66, 814 (2011).

22 L. Šebestíková, J. D’Hernoncourt, M.J.B. Hauser, S.C. Müller, A. De Wit, Phys. Rev. E 75, 026309 (2007).

23 R. Agrawal, D.H. West, V. Balakotaiah, Chem. Eng. Sci. 62, 4926 (2007).

24 D. Nguyen, V. Balakotaiah, Chem. Eng. Sci. 49, 3489 (1994).

25 A. De Wit, Phys. Rev. Lett., 87, 054502 (2001).

26 T. Kreis, J. Opt. Soc. Am. A 3, 847 (1986).

27 M. Takeda, H. Ina, and S. Kobayashi, J. Opt. Soc. Am. 72, 156 (1982).

28 J. D'Hernoncourt, A. Zebib and A. De Wit, Phys. Rev. Lett. 96, 154501 (2006).

29 J. D'Hernoncourt, A. De Wit, and A. Zebib, J. Fluid Mech. 576, 445 (2007).

30 T. Tóth, D. Horváth, Á Tóth, Chem. Phys. Lett. 442289 (2007).

31 C.T. Tan and G.M. Homsy, Phys. Fluids, 31, 1330 (1988).

32 J. Martin, N. Rakotomalala, D. Salin, Phys. Fluids 14902 (2002).

33 G. I. Sivashinsky, Comb. Sci. Tech. 15137 (1977). 